\title{
Anabases
}

ANABASES Traditions et réceptions de l'Antiquité

$15 \mid 2012$

Varia

\section{Bruno et Galilée face à l'infinité cosmique et à la relativité}

Jean Seidengart

\section{(2) OpenEdition}

Journals

Édition électronique

URL : http://journals.openedition.org/anabases/3757

DOI : 10.4000/anabases.3757

ISSN : 2256-9421

Éditeur

E.R.A.S.M.E.

Édition imprimée

Date de publication : 1 avril 2012

Pagination : 145-162

ISSN : 1774-4296

\section{Référence électronique}

Jean Seidengart, «Bruno et Galilée face à l'infinité cosmique et à la relativité », Anabases [En ligne], 15। 2012, mis en ligne le 01 avril 2015, consulté le 21 octobre 2019. URL : http://journals.openedition.org/ anabases/3757 ; DOI : 10.4000/anabases.3757 


\section{Bruno et Galilée face à l'infinité cosmique et à la relativité}

JeAn SEIDENGART

La CONJECTURE QUI ANIME notre propos, du moins dans le cadre de la science classique naissante, c'est qu'il était impossible de penser la relativitédu mouvement sans remettre en cause la conception finitiste du système du monde (comme ce fut le cas de l'héliocentrisme copernicien), car la preuve de sa finité reposait sur le mouvement diurne de la sphère des fixes. En outre, il était impossible de concevoir un univers infini contenant une pluralité innombrable de mondes finis sans relativiser toutes les déterminations d'ordre spatial ou temporel. En ce sens, nous voudrions montrer que c'est l'idée d'infini qui permettait, mieux que toute autre, de détruire toutes les déterminations absolues de la cosmologie antico-médiévale, tout en constituant le cadre idéal indispensable à l'établissement de la science classique. Sur ce point, nous rejoignons en partie la perspective traditionnelle des historiens des sciences établie depuis le milieu du XIX ${ }^{\mathrm{e}}$ siècle et dont Alexandre Koyré est l'un des derniers représentants.

Tout d'abord, nous nous proposons de cerner l'idée de relativité telle qu'elle est apparue initialement chez les Anciens, puis telle qu'elle fut remaniée par Copernic pour défendre et illustrer son système héliocentrique. Ensuite, nous analyserons comment elle fut reprise, élargie et transformée par Bruno pour donner lieu à la construction de sa cosmologie infinitiste. Enfin, nous tenterons de déterminer jusqu'à quel point la relativité fournit à Galilée le moyen de justifier le copernicianisme tout en le généralisant à l'instar de Bruno, mais pour des raisons liées à la mathématisation de la science du mouvement des graves. 


\section{Du relativisme à l'idée de relativité au sens de Copernic}

Il semble que la découverte du relatif soit liée à celle de la différence et de la variabilité des points de vue sur le monde, sur le cours de l'histoire, sur la valeur des choses, des actions et des connaissances. Les Anciens, déjà sensibles à cette variabilité des points de vue, l'avaient déjà dénoncé surtout au niveau des jugements de valeur, tel Héraclite qui disait : "Les cochons se complaisent davantage dans la fange que dans l'eau pure ${ }^{1}$."

Très tôt, les penseurs dits "socratiques " se sont efforcés de distinguer entre ce qui est en soi et ce qui est pour nous (comme entre l'invariant et le variable, ou l'absolu et le relatif) pour faire de la philosophie la quête des invariants et la recherche de l'en soi. Au contraire, les adversaires de Socrate restaient résolument attachés au relatif, à ce qui est pour nous, en arguant de notre impuissance à atteindre l'en soi des choses : telle était la thèse du sophiste Protagoras, que rapporte Platon dans le Théetète ${ }^{2}$. D'un côté, cette thèse de l'homme-mesure de toutes choses soulignait l'émergence de la subjectivité dans l'Antiquité, mais elle constituait aussi la matrice du scepticisme. En effet, suivant le pyrrhonisme, le célèbre argument du prós ti (c'est-à-dire de la relativité) déclarait impossible, par principe, l'accès à l'en soi des choses, en raison du caractère variable, instable et subjectif des impressions que produisent les objets empiriques sur nos divers sens. Ainsi, nous ne connaissons jamais les propriétés absolues des choses, mais seulement les impressions fugaces qu'elles produisent sur nous :

"Le mode selon le relatif (ho dè apò tôu prós ti), comme nous l'avons dit plus haut, est celui dans lequel l'objet réel apparaît tel ou tel relativement à ce qui le juge et à ce qui est observé conjointement, et sur ce qu'il est selon la nature nous suspendons notre assentiment (épéchomen) $)^{3}$."

"Ces trois modes [s.e. "celui d'après ce qui juge, celui d'après ce qui est jugé, celui qui vient des deux"] à leur tour se ramènent à celui du relatif (eis tòn prós ti), de sorte que le relatif (tòn prós ti) est le genre le plus haut, dont les trois nommés sont des espèces, auxquelles sont subordonnés les dix ${ }^{4}$."

Au moins, tous les Anciens étaient d'accord entre eux pour définir le relatif comme le fit Aristote qui écrivait dans son traité Des Catégories :

1 Héraclite, fragment DK, B, XIII ; tr. fr. Dumont, Delattre, in Les Présocratiques, Paris, Gallimard, 1988, Pléiade, p. 149.

2 Platon, Théétète, 152 a, Paris, Garnier-Flammarion, 1967 ; tr. Chambry, p. 73 : "Protagoras dit [...] que l'homme est la mesure de toutes choses. [...] Ne veut-il pas dire à peu près ceci, que telle elle est pour moi et que telle elle t'apparaît à toi, telle elle est aussi pour toi ?"

3 Sextus Empiricus, Esquisses pyrrhoniennes, I, 15, 167 ; tr. Pellegrin, Paris, Seuil, 1997, p. $140-143$.

$4 \quad$ Ibid. I, 14, 39 ; tr. Pellegrin, Paris, Seuil, 1997, p. 76-77. 
"On appelle relatives ces choses dont tout l'être consiste en ce qu'elles sont dites dépendre d'autres choses, ou se rapporter de quelque façon à autre chose ${ }^{5}$. "

Être relatif, c'est donc dépendre de quelque chose. Or, les discussions autour du relatif et de la relativité des phénomènes n'ont de sens qu'à partir de la distinction de l'en soi et du pour nous. Toutefois, si l'on redescend dans le monde des phénomènes et de l'observation courante, les catégories opératoires sont celles de l'observateur et de l'observé. Or, le phénomène où la relativité se manifeste de la manière la plus obvie, c'est bien celui du mouvement.

La relativité dite "optique " ou descriptive ${ }^{6}$ du mouvement était déjà bien connue depuis l'Antiquité : elle est même aussi ancienne que l'humanité. Mais, de tous les cas de relativité descriptive, c'est sûrement celui du bateau qui retint l'attention des Anciens dans la stricte mesure où le mouvement propre du bateau est en lui-même imperceptible (évidemment par brise légère, avec une mer calme et une vitesse stabilisée, etc.). D’où le fameux passage (qui n'est pas innocent) du De Revolutionibus de Copernic qui cite et commente un vers de Virgile :

"Pourquoi hésiterions-nous plus longtemps de lui [s.e. à la Terre] attribuer une mobilité s'accordant par sa nature avec sa forme, plutôt que d'ébranler le monde entier, dont on ignore et ne peut connaître les limites ? Et n'admettrions-nous pas que la réalité de cette révolution quotidienne appartient à la Terre, et son apparence seulement au ciel ! Et qu'il en est par conséquent comme lorsqu'Énée (chez Virgile) dit : "Nous sortons du port et les terres et les villes reculent." En effet, lorsqu'un navire flotte sans secousses, les navigateurs voient se mouvoir, à l'image de son mouvement, toutes les choses qui lui sont extérieures et, inversement, ils se croient être en repos, avec tout ce qui est avec eux. Or, en ce qui concerne le mouvement de la Terre, il se peut que c'est de façon pareille que l'on croit le monde entier se mouvoir autour [d'elle] ${ }^{7}$."

Cette métaphore du navire reçoit une signification cosmologique qui repose sur le principe épistémologique de la relativité descriptive du mouvement que Copernic avait défini préalablement au chapitre $\mathrm{V}$ de son De Revolutionibus, car il lui permet de montrer que le mouvement de la Terre est aussi plausible que celui de la sphère des fixes :

5 Aristote, Des Catégories, tr. fr., Paris, Vrin, rééd. 1997, p. 29.

6 Cette dénomination "relativité optique ", bien que traditionnelle chez les historiens des sciences, n'est pas tout à fait adéquate à ce qu'elle dénote : on devrait parler plutôt de relativité " descriptive ", comme le suggère très justement M.-A. TONNELAT in Histoire $d u$ principe de relativité, Paris, Flammarion, 1971, p. 22. Nous emploierons de préférence l'expression de "relativité descriptive " pour éviter toute équivoque.

7 Copernic, De Revolutionibus orbium coelestium, Nuremberg, 1543, tr. fr. partielle Koyré, Paris, Alcan, 1934 ; rééd. Blanchard, 1970, livre I, ch. VIII, p. 92-93. Ici, Copernic cite Virgile, Énéide, III, v. 72 : Provehimur portu, terraeque urbesque recedunt. 
"En effet, tout mouvement local apparent provient soit du mouvement de la chose vue, soit de celui du spectateur, soit d'un mouvement, inégal bien entendu, des deux. Car lorsque les mobiles (je veux dire : le spectateur et l'objet vu) sont animés d'un mouvement égal, le mouvement n'est pas perçu. Or, c'est de la Terre que ce circuit céleste est vu et représenté pour notre vision. Si donc quelque mouvement appartenait à la Terre, celui-ci apparaîtrait en toutes les choses qui lui sont extérieures, comme si elles étaient entrainées avec la même [vitesse], mais en sens contraire ; et telle est en premier lieu la révolution diurne ${ }^{8}$."

Sur ce point, Copernic s'était expressément appuyé sur un passage célèbre des Académiques de Cicéron qui nous apprend (en se référant à un passage de Théophraste ${ }^{9}$ ) que Hicétas de Syracuse faisait tourner la Terre sur elle-même mais qu'il immobilisait " le ciel, le soleil, la lune, les étoiles [...] car rien dans l'univers ne se meut à l'exception de la Terre ${ }^{10}$. " La suite du fragment de Cicéron contient une remarque témoignant d'une compréhension profonde de la relativité descriptive du mouvement, parce que c'est

" la Terre, qui en tournant (convertat) extrêmement vite sur son axe avec un mouvement de torsion (torqueat), produit les mêmes effets que si elle était fixe, le ciel restant immobile ${ }^{11}$."

Pour Copernic, cette remarque constituait une sorte de caution antique favorable à la réduction des apparences géostatiques par l'héliocentrisme copernicien :

«Partant de là, j’ai commencé, moi aussi, à penser à la mobilité de la Terre. Et quoique l'opinion semblait absurde, cependant, puis donc que je savais qu'à d'autres avant moi fut accordée la liberté d'imaginer n'importe quels cercles afin d'en déduire les phénomènes des astres, je pensais qu'il me serait également permis de faire l'expérience de rechercher si, en admettant quelque mouvement de la Terre, on ne pouvait trouver une théorie plus solide des révolutions des orbes célestes que ne l'étaient celles de ceux-ci ${ }^{12}$."

Cet argument de la relativité descriptive du mouvement implique la distinction entre un mouvement apparent et un mouvement réel, alors qu'il devient très difficile de dépasser le simple plan des apparences pour trancher de façon décisive entre l'apparence

8 Copernic, De Revolutionibus orbium coelestium, Nuremberg, 1543 ; tr. fr. Koyré, Paris, I, chap. V, p. 73-74.

9 Le passage en question de Théophraste se trouve in Phys. Opin., fr. 18, D. 492 ; cité par Cicéron, in Académiques, II, ch. XXXIX, $\$ 123$; tr. Kany-Turpin, Paris, GarnierFlammarion, 2010, p. 257.

10 Cicéron, Académiques, II, ch. XXXIX, $₫ 123$; tr. Kany-Turpin, Paris, GarnierFlammarion, 2010, p. 257-259.

11 Cicéron, op. cit., p. 259.

12 Copernic, De Revolutionibus orbium coelestium, Nuremberg, 1543, tr. fr. partielle Koyré, Paris, Alcan, 1934 ; rééd. Blanchard, 1970, Lettre-Préface, p. 44-45. 
et la réalité du mouvement. En effet, le risque de la relativité descriptive est de sombrer dans un nivellement général des apparences observationnelles qui alimenterait un scepticisme contre-productif pour la connaissance scientifique.

Copernic, bien au contraire, produisit une subversion profonde du concept de réalité, puisque c'est dans le détour par le théorique que surgit la «vraie » réalité (imperceptible de façon immédiate pour les sens), tandis que les apparences observationnelles sont proprement "réduites" au statut de pures illusions imputables à l'ignorance des véritables rapports entre l'observateur et l'observé. Désormais, grâce à la puissance de ses ressources théoriques, le sujet met préalablement en question les données observationnelles, afin de déjouer les pièges éventuels qu'introduirait nécessairement la méconnaissance (c'est-à-dire l'absence de toute prise en compte) de notre propre position d'observateurs terrestres et qui aurait pu immanquablement nous duper. On passe avec Copernic de l'apparence décrite " naïvement " à l'apparence déjouée ou réduite, c'està-dire reconduite aux raisons par l'effet desquelles l'apparence ne peut être autrement qu'elle n'est. Autant dire que Copernic sort, grâce aux ressources du théorique, de ce nivellement relativiste, ce qui lui confere une situation privilégiée, puisqu'il est à la fois dans le système du monde et hors de ce même système, indissociablement terrien et théoricien. Or, c'est précisément ce renversement des apparences qui fascina Giordano Bruno, parce qu'il trouvait, dans ce renversement opéré par Copernic, la confirmation d'une expérience de jeunesse qui le conduisit à renverser la cosmologie d'Aristote et à lui substituer un univers infini contenant une pluralité infinie de mondes finis.

\section{Relativité " descriptive " et relativité physique dans l'univers infini de Bruno}

Aristote avait fondé son géocentrisme et son finitisme cosmologique sur des arguments rationnels, mais en s'appuyant également sur les données observationnelles qu'il voulait toujours prendre en compte. Ainsi, contre l'idée d'une Terre en mouvement, Aristote remarquait, à propos de son éventuelle rotation axiale vers l'orient, que nous devrions voir, dans ce cas, les nuages et les oiseaux se diriger vers l'occident. De même, les mobiles lancés verticalement vers le haut ne pourraient jamais revenir à leur point de départ, pas plus qu'une pierre lâchée du haut d'une tour ne pourrait jamais tomber au pied de celle-ci, en raison de la grande vitesse de rotation de la Terre durant leur élévation et leur chute :

"On voit donc que c'est une nécessité manifeste pour la Terre d'être au centre et d'y rester immobile. Les raisons déjà données le prouvent, et, avec elles, le fait que les [corps] lourds lancés verticalement vers le haut retombent à leur point de départ, même si la force les projetait infiniment loin. D’après ces considérations, il est donc évident que la Terre 
n'est ni mue, ni située hors du centre. De notre exposé ressort en outre la raison de sa fixité $^{13}$."

Or, c'est justement pour réfuter cette objection aristotélicienne contre le mouvement de la Terre et pour prendre la défense de Copernic que Bruno a voulu approfondir la question de la relativité du mouvement et tenté de passer de la relativité descriptive à la relativité physique du mouvement. Dans ce but, il montre que : $1^{\circ}$ ) tout mobile appartient à un système (Bruno n'emploie pas le terme de référentiel) et possède le mouvement de son système d'appartenance (cf. le navire ou la Terre) ; $2^{\circ}$ ) c'est par rapport à son système d'appartenance que l'on peut parler du mouvement propre du mobile, sans pouvoir déterminer à l'intérieur de ce système si le système est lui-même en mouvement ou en repos, à condition que le navire ne prenne pas de gîte. Bien sûr, Bruno ne songe qu'à écarter les mouvements parasites du système sans thématiser la question de la vitesse uniforme ou stabilisée de son mouvement. Fort, ainsi, de cet argument qui relève d'une quasi-relativité physique, Bruno était certain de détenir une réponse suffisante à l'objection d'Aristote. D'où sa remarque :

" [Sмiтнo]. [...] il y a une différence entre le mouvement d'un navire et le mouvement de ce que le navire contient. S'il n'en était pas ainsi, on ne pourrait jamais traîner un objet en ligne droite d'un bord à l'autre du navire, quand celui-ci court au large ; ni retomber après un saut, à l'endroit même d'où l'on s'est élancé.

[Teofilo]. Ainsi se meut avec la terre tout ce qui se trouve sur la terre. Si donc, d'un point extérieur à la terre, on y jetait quelque objet, le mouvement terrestre l'empêcherait d'avoir une trajectoire droite ${ }^{14}$. "

De façon plus générale, c'est cet attachement aux fausses évidences sensibles caractéristique des philosophes péripatéticiens que dénonce Bruno, non pas en ce sens qu'il répudie les enseignements de la perception, mais parce que ceux-ci doivent être soumis au jugement de l'intellect pour déjouer les illusions des sens. Bruno écrit : "C'est à l'intellect (intelletto) qu'il convient de juger et de rendre raison des choses absentes et séparées par distance de temps et intervalle des lieux ${ }^{15}$. "Bruno part du fait que nos sens sont bornés parce qu'ils ne peuvent appréhender que les objets qui sont à leur portée, ce qui restreint considérablement la valeur de leurs informations :

"Les sens [...] confessent et rendent évidentes leur faiblesse et leur insuffisance en suscitant, avec leur horizon, une apparence de finitude dont la formation fait voir de

13 Aristote, Traité du ciel ; tr. fr. Paul Moraux, Paris, Belles Lettres, 1965, II, 14, 296 b 21-27, p. 97.

14 G. Bruno, La cena de le ceneri, 1584 ; tr. fr. Y. Hersant, Paris, Belles Lettres, 1994, Dialogo Terzo, cinquième proposition de Nundinio, p. 182-188.

15 G. Bruno, De l'infinito, 1584 ; tr. Cavaillé, Paris, Belles Lettres, 1995, p. 58-60. 
surcroît combien ils sont inconsistants. Or comme nous savons par expérience qu'ils nous trompent au sujet de la surface de ce globe où nous nous trouvons, à plus forte raison les devons-nous tenir pour suspects quant à ce terme qu'ils nous font concevoir dans la concavité stellifere ${ }^{16}$."

Plus encore que la relativité du mouvement, c'est la relativité des données observationnelles que Bruno analyse et qui l'ont conduit à l'infinitisation de l'univers, tandis que Copernic avait délibérément écarté cette question en la laissant expressément aux philosophes.

C'est précisément ce phénomène de l'horizon que Bruno analyse en évoquant un souvenir d'enfance avec émotion, dans un célèbre passage de son De immenso et innumerabilibus de 1591. Lorsqu'il observait le Vésuve avec ses yeux d'enfant depuis le haut du mont Cicala, près de Nola, sa ville natale, il croyait voir en lui le bout du Monde :

"C'est ainsi que dans mon enfance, j’ai cru qu'il n'y avait plus rien au-delà du Vésuve, sous prétexte qu'il n'y avait rien au-delà qui soit accessible à mes sens ${ }^{17}$. "

L'horizon semble enclore dans son confinement circulaire le monde perçu, et pourtant chacun de nos déplacements franchit cette limite illusoire qui n'a donc pas d'existence propre ou absolue. Il n'y a pas d'horizon en soi, mais toujours pour un observateur déterminé : c'est, en quelque sorte, le corrélat de la limitation de nos sens. Le phénomène d'horizon ne cesse d'accompagner les déplacements de l'observateur situant celui-ci immanquablement au centre de sa propre perspective. Ainsi, le confinement circulaire de l'horizon perceptif résulte bien de la projection de la finitude de nos sens, et non pas de la structure de l'Univers. L'expérience du phénomène d'horizon nous procure un double enseignement, car elle nous présente à la fois la détermination distincte d'une limite et le franchissement de cette limite suscitant ainsi en nous la pensée d'une " avancée au-delà » que rien ne peut réprimer ou contenir dans sa progression illimitée. Toutefois, cela ne signifie pas que pour Bruno l'idée d'un espace infini soit, en quelque sorte, comme l'aboutissement de cette progression illimitée de la pensée ou de l'imagination qui réitérerait indéfiniment son interrogation sur l'au-delà de la limite.

Au fond de la pensée brunienne il y a une intuition de l'infini qui ne parvient pas à s'expliciter entièrement dans une démonstration en forme : c'est l'intuition d'un Univers infini en acte, et c'est sur le fond (entendu également comme fondement) de cet infini extensif que se détache ou se découpe, pour lui, le confinement illusoire de l'horizon. Le franchissement de la limite ne fait que suggérer l'infini extensif qui le précède ontologiquement et logiquement. Puisque l'idée d'un monde clos confiné par une limite ultime et infranchissable est impensable sans contradiction, Bruno s'appuie

16 Ibid., Dialogo Primo, p. 60.

17 G. Bruno, De immenso et innumerabilibus, 1591, II, chap. VIII, in Op. lat., I, 1, éd. Fiorentino \& Tocco, 1879/1891, p. 285 ; cf. aussi Ibid., III, chap. I. C'est nous qui traduisons. 
sur son impensabilité à la fois pour incliner ses lecteurs vers l'infinitisme et pour réfuter tous les arguments finitistes.

Bruno a repris à Copernic : le renversement du système ptoléméen en faveur d'une astronomie héliocentrique ; le principe de relativité descriptive ; et, surtout, la réduction de l'illusion géocentrique, la première de toutes nos illusions.

Mais il ne faut pas oublier pour autant que Bruno rejette de l'œuvre de Copernic: son finitisme cosmologique, son système complexe d'épicycles et d'excentriques, ses sphères cristallines concentriques emboîtées les unes dans les autres, son culte de la sphéricité, sa conception d'un unique système solaire. Ainsi, Bruno a supprimé tout ce qui subsistait de l'astronomie alexandrine dans le système de Copernic, mais ce n'est pas pour des raisons " modernes ". En fait, il a détruit les restes d'aristotélisme que contenait encore le système copernicien. Bruno s'est appuyé sur la pensée des atomistes antiques pour critiquer et rejeter le finitisme cosmologique aristotélico-ptoléméen. Il a repris, notamment à Épicure et à Lucrèce, l'argument du bâton, de la main ou du javelot tentant de franchir les limites du Monde clos :

"Si quelqu'un étendait la main au-delà de cette convexité [de la sphère des fixes], cette main ne se trouverait pas en un lieu ; elle ne serait nulle part : et par conséquent elle n'aurait pas l'être. [...] J'ajoute aussi à cela qu'il n'y a point d'esprit qui ne conçoive la contradiction impliquée dans ce propos péripatéticien ${ }^{18}$. "

Il n'a pas échappé à certains commentateurs que cet argument épicurien selon lequel un trait lancé par une catapulte pourrait traverser les "murailles du monde » présentait une analogie avec l'axiome d'Eudoxe-Archimède. En définitive, cet argument très ancien montre l'impossibilité de penser et d'assigner une limite ultime (ou absolue) à l'univers, puisqu'il est de l'essence d'une limite d'introduire une séparation entre deux régions dans l'espace, et non pas à l'espace. En outre, tout ce qui viendrait limiter l'espace devrait à son tour être étendu, ce qui ne fait que reculer le problème. Du reste, la pensée brunienne se complaît à transgresser toutes les limites : aussi bien dans Aristote que dans Copernic. C'est dans cette expérience intellectuelle de l'impensabilité de toute limite ultime de l'univers que s'enracine l'infinitisme brunien. La métaphore de l'envol devient, elle aussi, un véritable procédé intellectuel et non pas une simple figure de style pour comprendre cette impensabilité :

"Voici alors apparaître l'homme qui a franchi les airs, traversé le ciel, parcouru les étoiles, outrepassé les limites du monde, dissipé les murailles imaginaires des première, huitième, neuvième, dixième et autres sphères qui auraient pu leur être ajoutées selon de vains mathématiciens et suite à l'aveuglement des philosophes vulgaires. [...] C'est lui qui avec les clefs de sa compétence a ouvert par ses recherches ceux des cloîtres de la vérité

18 Bruno, De l'infinito, 1584 ; tr. Cavaillé, Paris, Les Belles-Lettres, 1995, Dialogo Primo, p. 64. 
auxquels nous pouvions avoir accès. Il a mis à nu la nature, que des voiles enveloppaient ; il a donné des yeux aux taupes et rendu la lumière aux aveugles. [...] Notre raison n'est plus entravée par les fers des huit, neuf et dix mobiles imaginaires avec leurs moteurs. Nous le savons : il n'y a qu'un ciel, une immense région éthérée où les magnifiques foyers lumineux conservent les distances qui les séparent au profit de la vie perpétuelle et de sa répartition. Ces corps flamboyants, les astres, sont les messagers de la gloire et de la majesté suprêmes de Dieu. Ils se découvrent à nous comme l'effet infini d'une cause infinie, comme le vrai et vivant vestige d'une énergie infinie ${ }^{19}$."

L'infinité de l'Univers, c'est-à-dire de l'espace cosmique, devient la solution logique à ce problème :

"Ceci [c'est-à-dire un univers fini] est plus difficile à imaginer, que de penser l'univers infini et immense, car nous ne pouvons éviter le vide, si nous voulons poser l'univers fini $^{20}$."

Autrement dit, Bruno élargit et multiplie à l'infini le système global de Copernic. Chacune des "étoiles fixes " que nous percevons est, pour Bruno, un Soleil semblable au nôtre avec son cortège de planètes et de satellites; et il ne convient pas de se limiter aux étoiles visibles, mais Bruno pense en outre à l'infinité des étoiles dont nous ne pouvons percevoir qu'une quantité infime en raison de notre situation locale dans l'Univers. L'Univers infini de Bruno est constitué d'une infinité de mondes finis, véritable miroir de l'infinité divine. L'infinité de Dieu est, cependant, différente de l'infinité de l'Univers, mais seul un Univers infini peut convenir raisonnablement à l'infinie puissance, sagesse et bonté de Dieu comme son expression sensible : l'infinité de l'Univers est "l'effet infini de la Cause infinie".

Est-ce à dire que Bruno attribue l'infinité de manière équivoque à Dieu et à l'Univers ? Certes, il est clair que rien ne peut être extérieur à l'Univers infini, puisque son infinité implique précisément qu'il n'ait pas d'Autre. Le Dieu de Bruno se caractérise à la fois par son Unité et par son immanence totale au sein de l'Univers infini :

"Dieu est infini dans l'infini, partout en toutes choses, ni au-dessus ni à l'extérieur mais totalement intime à toutes choses ${ }^{21}$."

La théologie brunienne est solidaire de son ontologie et de sa cosmologie. L'infinité de l'Univers est comme dispersée dans cet "immense simulacre corporel ${ }^{22}$ "

19 Bruno, La cena de le ceneri, 1584 ; tr. fr. Y. Hersant, Paris, Les Belles Lettres, 1994, Dialogo Primo, p. 46-50.

20 Bruno, De l'infinito, 1584 ; tr. Cavaillé, Paris, Les Belles Lettres, 1995, Dialogo Primo, p. 66 .

21 Bruno, De immenso, 1591, Livre VIII, ch. X, in Op. lat., I, 2, p. 312. C'est nous qui traduisons.

22 Bruno, De immenso, Livre II, ch. XIII, in Op. lat., I, 1, p. 312. 
qui exprime et représente à sa manière l'infinité divine. L'univers, pourrait-on dire, participe de la perfection divine qui se reflète en lui, mais d'une façon seconde, dérivée, infiniment dispersée. D'autre part, chaque monde fini, chaque atome et chaque individu expriment respectivement $u n$ des aspects infinis de l'être infini ; mais chacun d'eux étant enclos dans sa détermination distincte, il ne peut à lui seul épuiser la variété infinie de la totalité de l'être. Dieu c'est donc l'Unité de l'Être qui "s'explique " à travers l'immensité cosmique et la multiplicité infinie des mondes innombrables, et qui se " complique " dès lors que l'esprit remonte de cette multiplicité infinie des êtres à leur "Cause, à leur Principe et à l'Un ». Bruno emploie ici la terminologie de l'explicatiocomplicatio qu'il emprunte à Nicolas de Cues, en lui rendant hommage. Pourtant, ce dernier ne cessait de clamer la transcendance de Dieu, alors que Bruno considère que Dieu est immanent à l'univers. Dieu assure l'Unité de l'Être, l'Uni-totalité de l'Univers, et finalement l'Unité de la pensée du Nolain dans l'immanence.

\section{De la relativité physique à la généralisation du copernicianisme chez Galilée}

Le copernicianisme de Galilée a pris ses distances avec la lettre même du De Revolutionibus de Copernic. Très tôt, Galilée rejeta l'existence de la sphère des fixes, à laquelle était resté très attaché le chanoine polonais, car il découvrit grâce aux observations du monde sidéral effectuées à l'aide de sa lunette que là où l'œil ne percevait aucun objet, son réfracteur pouvait en faire apparaître des myriades. En raisonnant par récurrence, Galilée pensait que si l'on pouvait se rendre sur l'une de ces étoiles (" télescopiques ", en langage moderne), il ne manquerait pas de nous apparaitre d'autres étoiles qui échappent aux lunettes situées sur la Terre en raison de leur trop grand éloignement. À partir du moment où les astronomes ont pu commencer à sonder quelque peu par des procédés optiques la troisième dimension du ciel, la sphère des fixes devint un simple moyen commode pour représenter la distribution des étoiles à partir d'un système de coordonnées angulaires équatoriales centré sur la Terre (ou sur le Soleil), mais elle finit par perdre toute réalité physique.

En outre, cette apparente sphéricité de la "voûte céleste " n'est simplement que la projection du mouvement diurne de la Terre sur le fond du ciel nocturne, donc une simple illusion dès que l'on se place dans une perspective copernicienne. D'où l'insistance avec laquelle Galilée s'est attaché à expliquer les raisons physiques de la relativité du mouvement. Il veut faire comprendre à Simplicio, le représentant du péripatétisme, qu'il est impossible de mettre expérimentalement en évidence le mouvement d'un système mécanique à l'intérieur dudit système, car il peut être imperceptible : ce qui rendait parfaitement plausible le système héliocentrique de Copernic. Pour montrer que le système héliocentrique est non seulement possible, mais aussi réel, Galilée a dû recourir à d'autres preuves (dont certaines sont d'ordre optique comme les phases de Vénus) sans détenir pour autant une preuve décisive. 
Par ailleurs, Copernic avait déjà pris expressément conscience que son géocinétisme soulevait nécessairement la question de savoir si l'Univers est fini ou infini. Or, cette question conduisait à mettre en doute l'existence de la sphère des fixes qu'il venait " d'immobiliser ». C'est précisément cette même question que souleva Galilée avec ses grands talents de polémiste dans son Dialogo de 1632, en insistant sur le fait que la preuve de la finité du cosmos aristotélicien était réduite à néant dans la perspective héliocentrique qui immobilise la sphère des étoiles fixes :

"Simplicio. - Si en effet le globe terrestre doit se mouvoir en un an sur la circonférence d'un cercle, c'est-à-dire sur le zodiaque, il ne peut pas en même temps être le centre de ce zodiaque ; or Aristote, Ptolémée et d'autres ont prouvé de bien des façons que la Terre est en ce centre. [...] Par centre, j'entends celui de l'Univers, celui du monde, celui de la sphère étoilée, celui du ciel.

SALViati. - Je pourrais très raisonnablement soulever la question de l'existence d'un tel centre dans la nature : ni vous ni personne n'a jamais prouvé que le monde est fini et possède une forme, ou bien qu'il est infini et sans terme. [...]

Simplicio. - Que le monde soit fini, limité et sphérique, Aristote le prouve par cent démonstrations.

SAlviati. - Mais elles se réduisent toutes à une seule qui, elle, se réduit à rien. Si en effet je nie son hypothèse, que l'Univers est mobile, alors toutes ses démonstrations s'écroulent, car il ne prouve la finitude et la limitation que d'un Univers mobile. Mais, pour ne pas multiplier ces disputes, je vous concède pour l'instant que le monde est fini et sphérique et qu'il a un centre ; puisque c'est de sa mobilité qu'on conclut à sa forme et à son centre. [...] Or, dites-moi, Signor Simplicio, si Aristote s'était trouvé contraint par des expériences très évidentes de changer en partie la disposition et l'ordre qu'il avait retenus pour l'Univers et d'avouer qu'il s'est trompé sur l'une de ces deux propositions - à savoir que la Terre est au centre et que la sphère étoilée se meut autour de ce centre -, lequel des deux aveux croyez-vous qu'il aurait choisi de faire ${ }^{23}$ ? "

Tandis que Copernic préférait conserver l'existence de la sphère des fixes, Galilée pour sa part la niait, du moins sur le plan physique. À cet égard, Galilée était plus proche de Bruno que de Copernic, bien qu'il n'existe absolument aucun passage de son œuvre ni de sa correspondance qui cite ou évoque la pensée du Nolain, même quand Kepler lui demande expressément son sentiment à ce sujet ${ }^{24}$. En effet, mieux valait être

23 Galilée, Dialogo sopra $i$ due massimi sistemi del mondo, 1632, Giornata terza, Opere, Ed. Nat., VII, p. 347-348 ; trad. fr. De Gandt, Paris, Seuil, 1992, p. 324-325.

24 Tel est bien le cas de la Dissertatio cum Nuncio Sidereo de Kepler qui pose des questions précises à Galilée à propos de Mélissos, Démocrite, Edmond Bruce et Giordano Bruno. 
prudent et faire silence à propos d'un auteur dont l'œuvre entière avait été mise à l'Index et qui a fini sur le bûcher de l'Inquisition. Galilée, à l'instar du Nolain, dénonçait le mouvement apparent de la "sphère des fixes " et l'existence même de cette sphère comme deux illusions du même ordre. Il est vrai que Bruno ne disposait pas des observations optiques de Galilée, mais il s'était appuyé sur les découvertes faites par Tycho Brahe au sujet des comètes et dont les conséquences venaient de "volatiliser " toutes les sphères cristallines emboîtées (encore chères à Copernic) à l'exception de la sphère des fixes. En revanche, Galilée, qui avait nié l'existence « supralunaire » des comètes, ne pouvait s'appuyer comme le Nolain sur les arguments de Tycho qu'il préférait ignorer purement et simplement. Puisque l'existence de la sphère "stellifere " n'était qu'une illusion pour Galilée, il lui fallait aussi écarter la notion tenace de centre de l'univers et, par la même occasion, toute la cosmologie péripatéticienne traditionnelle, comme il le rappelle dans une lettre au savant aristotélicien Fortunio Liceti :

"Le problème du centre de l'Univers et la question de savoir si la Terre y est située sont des moins considérables en astronomie, attendu que les meilleurs astronomes se contentent de supposer que le globe terrestre est d'une grandeur infime en comparaison de l'orbe étoilé et, quant à sa situation, qu'il se trouve, ou au centre de la révolution diurne de cet orbe, ou séparé dudit centre par une distance négligeable. Mieux vaut donc s'épargner l'illusion de pouvoir démontrer, ou que les étoiles fixes sont logées dans un espace circonscrit par une surface sphérique, ou qu'elles sont situées en tel ou tel lieu et immédiatement éloignées les unes des autres. Aussi bien, vouloir assigner un centre à cet espace dont on ne sait et dont on ne peut savoir quelle est sa forme ni même s'il a forme et figure, c'est là, à mon sentiment, une entreprise oiseuse et vaine ; et croire que la Terre est constituée en un centre dont on ne sait s'il existe au monde est, comme je l'ai dit, une entreprise décevante ${ }^{25}$."

Du reste, l'idée de l'incommensurabilité des dimensions du globe terrestre avec celles de la sphère des fixes était déjà communément reçue chez les astronomes formés à l'École de Ptolémée. C'est d'ailleurs ce qu'enseignait Galilée lui-même dans son manuel de cosmographie (encore entièrement ptoléméen) : le Trattato della Sfera de 1597. Ainsi, pour démontrer que « le globe terrestre est une masse insensible en comparaison du Ciel ", Galilée s'appuyait sur quatre arguments dont l'un des plus convaincants, qui figure aussi dans l'Almageste de Ptolémée, faisait valoir que si la Terre possédait des dimensions notables par rapport au Ciel, " nous ne pourrions voir la moitié de

Dans ses réponses Galilée ne dit mot sur le Nolain : cf. Opere, Ed. Nat., III, p. 106-123 et X, p. 320-335.

25 GaliléE, Lettre à Fortunio Liceti de janvier 1641, Opere, Ed. Nat., XVIII, p. 293-294; tr. fr. P. H. Michel in Galilée : Dialogues et Lettres choisies, Paris, Hermann, 1966, p. 428. 
celui-ci, mais une portion nettement inférieure ${ }^{26}$ ». Or, la suite du texte de la lettre à Fortunio Liceti, qui évoquait l'éventualité copernicienne d'une Terre orbitant autour du Soleil, montrait que la distance de la Terre au Soleil (donc, le rayon de l'orbite terrestre annuelle) est également une grandeur insensible par rapport aux dimensions de la sphère stellifere. Ce qui revient à dire qu'il est stérile de chercher à situer le centre d'une sphère dont on ignore les dimensions, parce que le rayon de la Terre, aussi bien que le rayon de son orbite annuelle, ne constituent pas des bases suffisamment grandes pour trianguler une distance stellaire. Dans ce cas, la trigonométrie ne nous est d'aucun secours, puisque nous ne disposons d'aucune parallaxe stellaire annuelle. Tycho Brahe le savait fort bien, et c'est d'ailleurs l'une des raisons qui l'avaient conduit à abandonner le système copernicien pour établir à sa place un système géo-héliocentrique. Galilée, pour sa part, préférait abandonner l'existence de la sphère des fixes pour conserver l'Univers déjà "immensément " agrandi par Copernic. Mais, contrairement à Copernic, Galilée laissait ouverte la question de savoir si l'Univers possède ou non une forme ; c'est même pour cette raison qu'il préférait parler d' "espace ${ }^{27}$ " (spazio) plutôt que de ciel, puisque la voûte apparente de celui-ci n'est désormais qu'un effet de perspective dû à notre situation locale d'observateurs terrestres.

En outre, Galilée affirmait, tout comme le fit Bruno dès 1584, que toutes les étoiles sont des Soleils en tous points semblables au nôtre, ce qui n'exclut nullement qu'elles puissent être à leur tour le centre de systèmes planétaires comparables à notre système solaire. Galilée considérait ainsi que l'univers comprend en lui une pluralité innombrable de systèmes coperniciens, bien qu'il ne puisse expliquer comment les étoiles fixes peuvent demeurer en suspens dans l'espace cosmique :

"Vous voyez aussi, dit Salviati, combien il convient mieux d'ôter à l'Univers le mouvement vertigineux en vingt-quatre heures et de permettre aux étoiles qui sont autant de Soleils de jouir comme notre Soleil d'un perpétuel repos. Voyez comme il est facile, dans cette première ébauche, de rendre raison des apparences si imposantes des corps céles$\operatorname{tes}^{28}$."

26 GaLILÉE, Trattato della Sfera ovvero Cosmografia, 1597, in Opere, Ed. Nat., II, p. 221-222: Che la Terra sia d'insensibil grandezza in comparazione del cielo. Esser di mole insensibile il globo terrestre paragonato col cielo, lo demostrano... ragioni... La prima è, che se la terra fusse di notabil grandezza rispetto alla sfera stellata, noi non potremmo vedere la metà del cielo, ma parte sensibilmente minore... Notons, toutefois, que cet argument avait permis à Galilée d'affirmer que la Terre était au centre de l'Univers (ce qu'il ne croyait nullement depuis au moins deux ans).

27 Les termes originaux de GALILÉE sont les suivants : Parimente il voler assegnar centro a quello spazio che non si sa nè si puo sapere quale sia la sua figura, nè pure se egli di qualche figura sia figurato, è impresa, al mio parere, supervacanea e vana. Cf. Opere, Ed. Nat., XVIII, p. 293-294.

28 Galilée, Dialogo sopra i due massimi sistemi del mondo, 1632, Giornata terza, Opere, Ed. Nat., VII, p. 354 ; trad. fr. De Gandt, Paris, Seuil, 1992, p. 330 : Vedete poi quanto 
Ce qui nous importe le plus ici, c'est que Galilée ait admis non seulement l'immensité de l'espace cosmique, mais aussi l'existence d'une quantité innombrable d'étoiles semblables à notre Soleil. Certes, on pourrait objecter que ce n'est là qu'une façon de parler et de laisser ouverte la question de la finité ou de l'infinité de l'Univers en se gardant bien (au moins par prudence) d'essayer de la trancher. En fait, si l'on examine soigneusement le texte du Dialogo, on découvre un passage de la Giornata terza qui a des résonances fortement bruniennes (comme l'a remarqué justement M. Clavelin ${ }^{29}$ ), tant sur le plan du vocabulaire que de l'argumentation, en ce qu' elle relativise les termes de grand et de petit :

"Je dis que grand, petit, immense, très petit (grande, piccolo, immenso, minimo) etc., ne sont pas des termes absolus, mais relatifs ; la même chose, quand on la compare à des choses différentes, pourra être dite tantôt immense, tantôt plus que petite, imperceptible. Dans ces conditions, je demande par rapport à quoi la sphère de Copernic est déclarée trop vaste. À mon avis, on ne peut la comparer et la dire trop vaste que par rapport à une autre chose du même genre. [...] Si à chaque fixe on devait attribuer pour la contenir autant d'espace dans le monde [s. e. que les sphères de Jupiter et de Saturne], il faudrait que l'orbe qui contiendrait leur multitude innombrable soit des milliers et des milliers de fois plus grand que celui qui suffit à Copernic. Ne dites-vous pas aussi d'une étoile fixe qu'elle est très petite (et même je veux parler des étoiles les plus visibles et non de celles qui échappent à notre vue) ? Si on les dit très petites, c'est par rapport à l'espace qui les entoure. Si toute la sphère étoilée n'était qu'un seul corps brillant, qui ne comprendra que, dans l'espace infini (nello spazio infinito), on peut assigner une distance assez grande pour que cette sphère lumineuse paraisse aussi petite, et même plus, qu'une étoile fixe vue maintenant de la Terre ? On considérerait donc comme petit ce qu'actuellement on dit immensément grand ${ }^{30}$."

Tout d'abord, cette relativisation des termes de grand et de petit, d'immense et de minimum est l'un des thèmes essentiels de Bruno pour anéantir l'absoluité des déterminations cosmologiques qu'impliquait immanquablement le finitisme aristotélicien. Cependant, malgré ces ressemblances frappantes entre les propos de Galilée et ceux du

acconciamente vien levato dall'universo il precipitosissimo moto delle 24 ore, e come le stelle fisse, che sono tanti Soli, conforme al nostro Sole godono una perpetua quiete. Vedete in oltre quanta agevolezza si trovi in questo primo abozzamento, per render le ragioni di apparenze tanto grandi ne' corpi celesti. C'est nous qui soulignons.

29 M. Clavelin, La philosophie naturelle de Galilée, Paris, Colin, 1968, II partie, p. 216 et note 101 : "D'où l'inclination dont Galilée témoigne à certains moments pour une interprétation infinitiste, soit qu'il compare comme Bruno les étoiles à autant de Soleils, soit qu'il évoque ce point de "l'espace infini" d'où la sphère des fixes de l'astronomie ptoléméenne ne paraît pas plus grande qu'une seule étoile vue de la Terre. »

30 Galilée, Dialogo sopra i due massimi sistemi del mondo, 1632, Giornata terza, in Opere, Ed. Nat., VII, p. 396-397 ; trad. fr. De Gandt, Paris, Seuil, 1992, p. 364-365. 
Nolain, leurs intentions respectives les séparent ici profondément. Tandis que Bruno s'installait d'emblée dans une perspective infinitiste à partir de laquelle il montrait les inconséquences du finitisme cosmologique d'Aristote, Galilée ne considérait nullement l'infinité de l'univers comme une évidence première, mais comme une simple quaestio disputata parmi d'autres. De plus, Galilée avait écarté l'idée naïve des péripatéticiens d'après laquelle tout devrait être ordonné en fonction de la Terre et de l'homme. D'ailleurs, l'ignorance où nous sommes de l'utilité que peuvent présenter les planètes lointaines, les distances interstellaires énormes et l'immensité de l'Univers copernicien ne sauraient être un argument suffisant pour les récuser! Galilée a même décuplé la puissance de cet argument en soulignant que si l'univers de Copernic est bien plus grand que celui d'Aristote et de Ptolémée, on ne peut s'empêcher d'en concevoir un autre qui soit encore incomparablement plus grand! En effet, chacune des étoiles visibles à l'œil nu représente pour Galilée autant de systèmes coperniciens avec leurs planètes respectives, sans oublier les étoiles innombrables qui n'apparaissent qu'à la lunette, car « il y en a cent fois autant que nous ne pouvons voir ${ }^{31}$ ». Bref, cet accroissement incommensurable des dimensions de l'univers anéantit l'idée qu'un ordre cosmique implique nécessairement un système clos où toutes ses parties doivent être justement proportionnées entre elles. C'est la voie ouverte au thème du ciron avant Pascal, à celui des Voyages de Gulliver avant Swift et à celui de Micromégas avant Voltaire. Toutefois, s'il est vrai que dans l'infini les notions de plus grand, de plus petit, d'égal et d'inégal perdent toute leur pertinence, est-ce à dire que Galilée soit passé dans le camp infinitiste en sautant par-dessus l'abîme qui sépare conceptuellement l'immensité de l'infinité proprement dite?

Certes, Galilée n'ignorait pas les paradoxes qu'implique la notion d'infini si on l'emploie sans précautions, car il avait commencé à rédiger dès 1610 un ouvrage sur le problème de la "composition du continu » qui ne vit jamais le jour, mais qui fut réutilisé dans ses Discorsi e dimostrazioni matematiche de 1638. Loin de se complaire dans ces paradoxes, Galilée s'était soucié de montrer qu'il existe une différence radicale entre les propriétés du fini et de l'infini, d'où il suit que tous les paradoxes de l'infini naissent justement de l'ignorance de cette différence radicale. Donc, si l'on veut échapper à toutes les difficultés, apories et absurdités qui accompagnent l'idée d'infini, il faut exclure du traitement de l'infini tous les prédicats qui ne conviennent qu'au fini, comme le nombre, la mesure et surtout les concepts comparatifs d'égalité et d'inégalité, de plus grand et de plus petit.

Les Anciens avaient préféré éliminer l'infini (en le contournant à l'aide d'expédients potentialistes ${ }^{32}$ ) pour sauvegarder la logique traditionnelle, tandis que Galilée

31 Galilée, op. cit., Giornata Terza, Opere, Ed. Nat., VII, p. 398 ; trad. fr. De Gandt, Paris, Seuil, 1992, p. 366 : E cento volte tante ve ne sono del tutto a noi invisibili.

32 Je pense ici, par exemple, au concept de quantité " aussi grande que l'on voudra ". Ainsi, Aristote concluait dans son étude du concept d'infini, in Physique, III, 7, 207b 2-13, tr. 
pour sa part avait préféré limiter le champ d'application légitime de celle-ci pour ménager une place à l'infini. En effet, puisque notre entendement est fini, nous ne disposons que d'une approche négative de l'infini. Pour éviter les paradoxes de l'infini, il faut nous abstenir : $1^{\circ}$ ) de comparer deux infinis et $2^{\circ}$ ) de comparer le fini et l'infini. Si nous respectons cette limitation dans l'emploi de nos facultés cognitives, plus aucune absurdité ne saurait désormais venir miner notre réflexion sur l’infini :

"SAlviati : Ainsi, de votre ingénieux discours, il résulte que le plus grand, le plus petit et l'égal sont des attributs inapplicables non seulement aux infinis, mais même aux infinis et aux finis comparés entre eux ${ }^{33}$."

En somme, Galilée est prêt à admettre l'existence de quelque chose qui échappe à la puissance de notre faculté de comprendre, alors que pour le péripatéticien Simplicio il suffit qu'une notion nous soit incompréhensible pour que nous soyons assurés de sa non-existence. Simplicio faisait de l'esprit humain la mesure de ce qui est, de ce qui peut être et de ce qui n'est pas. Pour Galilée, c'est Dieu seul, dont la puissance et l'omniscience sont infinies, qui peut vraiment décider de ce qui est ou de ce qui n'est pas ${ }^{34}$. Tel était bien le nouvel état d'esprit propre à Galilée lorsqu'il en vint à se demander si l'univers est fini ou infini.

Nombre de commentateurs furent frappés par l'extrême prudence de Galilée sur la question de l'infinité de l'univers. Il est vrai que l'observation ne permet nullement de trancher en faveur du fini ou de l'infini. La lunette montre seulement qu'il existe bien plus d'étoiles que ce que nos yeux nous permettent d'apercevoir : notamment la profusion incroyable d'étoiles que contient la Voie lactée. La lunette de Galilée lui donna l'impression que le monde sidéral était dépourvu de forme propre et que l'espace est d'une profondeur insondable, comme il le confia à Francesco Ingoli, le Secrétaire de la Congrégation De propaganda fide :

Carteron, Paris, Belles Lettres, t. 1, p. 107-108 : «Les considérations des mathématiciens, en supprimant l'infini qui existerait en acte dans le sens de l'accroissement, [...] n'ont pas besoin et ne font point usage de l'infini, mais seulement de grandeurs aussi grandes qu'ils voudront, mais limitées. »

33 GALILÉE, Discorsi e dimostrazioni matematiche intorno a due nuove scienze attenenti alla meccanica, Leyde, 1638, ed. Nat., VIII, p. 79-80 ; tr. fr. P. H. Michel in Galilée : Dialogues et Lettres choisies, Paris, Hermann, 1966, p. 258.

34 Galilée, Dialogo, 1632, Giornata terza, Opere, Ed. Nat., VII, p. 397 ; trad. fr. De Gandt, Paris, Seuil, 1992, p. 365 : «SAGREDO - Quelle énorme ineptie : ils [s. e. les Péripatéticiens] veulent que Dieu ait fait l'Univers proportionné à la faible capacité de leur raison plutôt qu'à sa puissance immense et même infinie !» 
" $\mathrm{Ni}$ vous ni personne d'autre au monde ne sait ni ne peut humainement savoir non seulement quelle est la figure [de l'Univers], ni même s'il possède une quelconque figure $^{35}$."

Galilée était prêt à admettre l'éventualité que l'Univers soit infini, puisqu'il savait qu'il venait de détruire le principal argument de la cosmologie traditionnelle en faveur du fini en adoptant le système héliocentrique qui immobilise l'armée des étoiles fixes. En effet, pour Aristote, ce qui est infini ne peut tourner ; or, il est manifeste que le ciel tourne, donc il est limité. Or, Galilée, à la suite de Copernic, attribuait à la Terre le mouvement apparent du ciel, ce qui réduit à néant l'option finitiste d'Aristote.

En outre, une lettre de Galilée à Fortunio Liceti présentait un tout nouvel argument assez original en faveur d'une conception infinitiste de l'univers qui s'appuyait sur la théorie de la connaissance :

"Les raisons qui furent alléguées en faveur d'un parti ou de l'autre [sur la grandeur finie ou infinie de l'Univers] sont très subtiles, mais dans mon cerveau ni les unes ni les autres n'aboutissent à des conclusions nécessaires, en sorte que je reste dans le doute en me demandant laquelle de ces deux assertions est vraie. Toutefois, un seul argument, qui m'est personnel, m'incline plutôt vers l'infini que vers le fini (un solo mio particolare discorso m'inclina più all'infinito che al terminato), vu que je ne me l'imagine ni ne peux me l'imaginer ni limité ni illimité et infini. Et puisque l'infini, en raison de sa nature propre, ne peut être compris par notre intellect limité, ce qui ne se produit pas dans le cas de ce qui est fini et enclos à l'intérieur d'une limite, je dois référer mon incapacité de comprendre plutôt à l'infinité qu'à la finité dans laquelle il n'y a pas à chercher de raison pour son incompréhensibilité. Mais c'est là une de ces questions, comme l'affirme volontiers votre Excellence, heureusement inexplicables par des raisonnements humains, et peut-être semblables à celles qui ont trait à la prédestination, au libre-arbitre et aux autres où seules les Pages Sacrées et les divines assertions peuvent pieusement nous apaiser ${ }^{36}$. "

L'argument, si l'on peut s'exprimer ainsi, ne vaut pas kat'alètheian mais seulement kat'anthrôpon, c'est-à-dire qu'il ne tranche pas selon la vérité de la chose, mais uniquement par rapport aux moyens limités dont nous disposons pour appréhender celle-ci. Si nous suivons de très près le texte, nous constatons que Galilée suit une démarche apagogique, c'est-à-dire par l'absurde. En effet, il pose qu'il lui est également impossible d'imaginer un univers fini et un Univers infini. Cela signifie que l'infini échappe par définition, ou par principe, aux capacités limitées de notre imagination et de notre entendement. En revanche, ce qui est moins évident, c'est que Galilée ne se puisse représenter un univers fini : peut-être parce qu'il peut toujours se figurer

35 Lettre de GaliléE à Ingoli de 1624, in Opere, Ed. Nat., VI, p. 518 : Nè voi nè altro uomo del mondo sappia o possa umanamente sapere, non solo qual sia la figura sua, ma se egli ha figura veruna.

36 Galilée, lettre à Fortunio Liceti du 24 septembre 1639, Opere, Ed. Nat., XVIII, p. 106. 
d'autres étoiles au-delà de ses limites, comme on vient de le voir. Bien qu'il ne nous donne aucune indication supplémentaire, il semble que l'idée d'un univers fini, mais qui ne serait pourtant limité par rien, soit contradictoire. Raisonnant par l'absurde, Galilée montre seulement que si l'Univers était fini, son entendement également limité devrait pouvoir le comprendre, ce qui n'est pas le cas. Il ne reste plus alors qu'une seule possibilité logique : il est donc tout à fait compréhensible que l'incompréhensibilité de l'Univers soit due à son infinité, attendu que l'infinité est par nature incompréhensible à un entendement fini. L'argument de Galilée, de forme purement négative, a réussi à surmonter la balance entre le fini et l'infini tout en débouchant sur une sorte de « docte ignorance ». Que l'infini reste pour nous incompréhensible, cela ne saurait exclure que l'univers soit en lui-même infini.

Contrairement à Bruno, Galilée ne prétend nullement que l'infini soit accessible en quelque manière à notre esprit fini, mais il admet volontiers que ce qui nous dépasse (comme l'idée d'infini) puisse exister. Mais, absolument parlant, Galilée confesse prudemment que ce problème de l'infinité cosmique restera à jamais insoluble pour l'humanité et qu'il est donc préférable de s'en remettre sur ce point aux enseignements de la révélation. C'est une attitude fort habile pour la tranquillité personnelle du savant, mais on peut légitimement se demander si la révélation apporte une réponse. Si l'on en croit l'Évêque Tempier avec ses interdits de 1277, l'Écriture et la théologie nous enjoignent de croire, contrairement à ce que prétendaient les Péripatéticiens médiévaux, que Dieu, en vertu de sa toute-puissance, a pu créer une infinité de mondes. En se déclarant soumis à l'autorité de l'Église et de l'Écriture, Galilée en revenait, ironiquement, à l'argument de Sagredo suivant lequel : « Dieu a fait l'Univers... proportionné à sa puissance immense et même infinie. "Sur ce point, Galilée rejoint la position de Bruno, mais pour de toutes autres raisons.

Jean SEIDENGaRT

Professeur de philosophie
Université Paris Ouest-Nanterre.
jean.seidengart@sfr.fr
jean.seidengart@u-paris10.fr

